\title{
Antiretroviral penetration into the CNS and incidence of AIDS-defining neurologic conditions
}

Ellen C. Caniglia Lauren E. Cain, PhD Amy Justice, $\mathrm{PhD}$ Janet Tate, PhD Roger Logan, PhD Caroline Sabin, PhD Alan Winston, MD Ard van Sighem, PhD Jose M. Miro, PhD Daniel Podzamczer, PhD Ashley Olson, PhD José Ramón Arribas, MD Santiago Moreno, $\mathrm{PhD}$ Laurence Meyer, PhD Jorge del Romero, MD François Dabis, PhD Heiner C. Bucher, MD Gilles Wandeler, MD Georgia Vourli, MSc Athanasios Skoutelis, MD Emilie Lanoy, PhD Jacques Gasnault, MD Dominique Costagliola, $\mathrm{PhD}$

Miguel A. Hernán, DrPH On behalf of the HIVCAUSAL Collaboration

Correspondence to Ellen C. Caniglia: ecanigli@hsph.harvard.edu

Editorial, page 109

Supplemental data at Neurology.org

\section{ABSTRACT}

Objective: The link between CNS penetration of antiretrovirals and AIDS-defining neurologic disorders remains largely unknown.

Methods: HIV-infected, antiretroviral therapy-naive individuals in the HIV-CAUSAL Collaboration who started an antiretroviral regimen were classified according to the CNS Penetration Effectiveness (CPE) score of their initial regimen into low (<8), medium (8-9), or high (>9) CPE score. We estimated "intention-to-treat" hazard ratios of 4 neuroAIDS conditions for baseline regimens with high and medium CPE scores compared with regimens with a low score. We used inverse probability weighting to adjust for potential bias due to infrequent follow-up.

Results: A total of 61,938 individuals were followed for a median (interquartile range) of 37 $(18,70)$ months. During follow-up, there were 235 cases of HIV dementia, 169 cases of toxoplasmosis, 128 cases of cryptococcal meningitis, and 141 cases of progressive multifocal leukoencephalopathy. The hazard ratio (95\% confidence interval) for initiating a combined antiretroviral therapy regimen with a high vs low CPE score was $1.74(1.15,2.65)$ for HIV dementia, $0.90(0.50,1.62)$ for toxoplasmosis, $1.13(0.61,2.11)$ for cryptococcal meningitis, and 1.32 $(0.71,2.47)$ for progressive multifocal leukoencephalopathy. The respective hazard ratios $(95 \%$ confidence intervals) for a medium vs low CPE score were $1.01(0.73,1.39), 0.80(0.56,1.15)$, $1.08(0.73,1.62)$, and $1.08(0.73,1.58)$.

Conclusions: We estimated that initiation of a combined antiretroviral therapy regimen with a high CPE score increases the risk of HIV dementia, but not of other neuroAIDS conditions. Neurology ${ }^{\circledR}$ 2014;83:134-141

\section{GLOSSARY}

CART $=$ combined antiretroviral therapy; $\mathbf{C I}=$ confidence interval; $\mathbf{C P E}=$ CNS Penetration Effectiveness; ICD-9 $=$ International Classification of Diseases, ninth revision; NNRTI = nonnucleoside reverse transcriptase inhibitor

AIDS-defining neurologic disorders, or neuroAIDS, include HIV dementia and the opportunistic infections toxoplasmosis, cryptococcal meningitis, and progressive multifocal leukoencephalopathy. ${ }^{1-3}$ The incidence of neuroAIDS in developed countries decreased after the introduction of combined antiretroviral therapy (cART) in $1996,{ }^{2,4-9}$ but antiretroviral drugrelated neurotoxicity remains a concern. ${ }^{2,10-12}$

The risk of neuroAIDS may depend on the concentration of antiretrovirals in the CNS, which is a function of their ability to penetrate the blood-brain barrier. Greater exposure of antiretrovirals in the CNS may decrease the HIV RNA in the CSF, ${ }^{13}$ but may also be neurotoxic. ${ }^{14,15}$ One proposed method to assess a drug's penetrative ability into the CNS is via the CNS Penetration Effectiveness (CPE) ranking system. In cohort studies to date, lower CPE ranks are associated with higher CSF HIV RNA after adjusting for a number of clinical variables. ${ }^{13,16-18}$

While the association between CPE rank and CSF HIV RNA is reported, the connection between CPE rank and clinical outcomes remains unclear. ${ }^{17,19-22} \mathrm{~A}$ randomized controlled trial comparing a CNS-targeted therapy to a non-CNS-targeted therapy among 49 individuals with HIV-associated neurocognitive disorders found no difference in improvement of neurocognitive

Authors' affiliations are listed at the end of the article.

Contributors to the HIV-CAUSAL Collaboration are listed on the Neurology ${ }^{\circledR}$ Web site at Neurology.org.

Go to Neurology.org for full disclosures. Funding information and disclosures deemed relevant by the authors, if any, are provided at the end of the article. 
performance after 16 weeks. ${ }^{23}$ An observational study in the United Kingdom found a similar incidence of neuroAIDS in individuals with high and low CPE scores. ${ }^{24}$

Herein, we present estimates of the effect of CPE score on the incidence of 4 neuroAIDS conditions among individuals with HIV-1 infection included in a large multinational collaboration of cohort studies from Europe and the United States.

METHODS Standard protocol approvals, registrations, and patient consents. Research using the HIV-CAUSAL Collaboration was determined to be nonhuman subjects research by the Institutional Review Board of the Harvard School of Public Health because it involves the study of existing data that are analyzed in such a manner that the subjects cannot be identified, as set forth in US federal regulations. Written informed consent from patients was not required because all data were completely anonymized.

Study population. The HIV-CAUSAL Collaboration includes prospective cohort studies from 6 European countries and the United States. All cohorts included in the HIV-CAUSAL Collaboration were assembled prospectively and are based on data collected for clinical purposes from national health care systems that offer universal access to care. Each cohort in the collaboration collected data prospectively, including all CD4 cell counts, HIV RNA measurements, treatment initiations, deaths, and AIDS-defining illnesses (including the events of interest: HIV dementia, toxoplasmosis, cryptococcal meningitis, and progressive multifocal leukoencephalopathy).

The individual cohort studies used in these analyses are UK CHIC (United Kingdom), ATHENA (the Netherlands), FHDHANRSCO4 (France), SHCS (Switzerland), PISCIS (Spain), CoRIS/ CoRIS-MD (Spain), VACS-VC (US veterans), AMACS (Greece), and AQUITAINE (France). Four cohorts of seroconverters with relatively high $\mathrm{CD} 4$ counts did not have any neuroAIDS events and were excluded from the analyses.

We restricted our analyses to individuals with HIV-1 infection who met the following criteria at baseline (starting in January 1998): age 18 years or older, no history of AIDS (defined as the onset of any category C AIDS-defining illness), ${ }^{25}$ antiretroviral therapy naive (as defined elsewhere ${ }^{26}$ ), no pregnancy (when information was available), CD4 cell count and HIV RNA measured within the previous 6 months, and initiating a complete antiretroviral regimen (see below) consisting only of drugs with known CPE ranks.

We conducted separate analyses for the following neuroAIDS events: HIV dementia, toxoplasmosis, cryptococcal meningitis, or progressive multifocal leukoencephalopathy. Non-Hodgkin lymphoma was not included as an event because we could not differentiate primary brain lymphoma from other types of non-Hodgkin lymphoma. We also looked at a combined endpoint of any of the 3 opportunistic infections (toxoplasmosis, cryptococcal meningitis, or progressive multifocal leukoencephalopathy). The date of neuroAIDS was identified by the treating physicians. One of the contributing cohorts (VACS) used ICD-9 codes to identify incident neuroAIDS cases. The other contributing cohorts used diagnostic procedures that reflect standard clinical practice in Europe rather than standardized research criteria.

For each patient, follow-up started on the date of initiation of a complete antiretroviral regimen-defined as treatment with at least 2 nucleoside reverse transcriptase inhibitors plus either one or more protease inhibitors, one or more nonnucleoside reverse transcriptase inhibitors (NNRTIs), one entry/fusion inhibitor, or one integrase inhibitor-and ended at death, 12 months after the most recent laboratory measurement, pregnancy (if known), the cohort-specific administrative end of follow-up (ranging between December 2003 and February 2013) or the event of interest, whichever occurred earlier.

Assessment of antiretroviral CNS exposure. The $2010 \mathrm{CPE}$ ranking system is a proposed method for measuring the penetrative ability of different antiretroviral drugs into the CNS. Each drug is given a rank ranging from 1 to 4 based on pharmacokinetic and pharmacodynamic data, drug characteristics, results of clinical studies, and effectiveness in reducing CSF viral load or improving cognition. A rank of 4 represents the best penetration or effectiveness. ${ }^{14,16}$ The CPE score for a given regimen is calculated by summing the ranks of each drug in the regimen. We categorized the CPE score for a regimen as low $(<8)$, medium (8-9), or high $(>9)$ based on the distribution of the data (the cut points were approximately at the median and the 75 th percentile) (figure 1). Because our estimates may be sensitive to the chosen cut points, we also treated the CPE score as a continuous variable.

Statistical methods. Using a pooled logistic regression model, we estimated the average "intention-to-treat" neuroAIDS hazard ratio for a high and a medium baseline CPE score compared with a low baseline CPE score. Under the assumption that the monthly probability of an event is small (a condition satisfied in our study), the parameters of our pooled logistic model closely approximate the parameters of a Cox proportional hazards model. ${ }^{27}$ We computed these estimates separately for each of the 4 neuroAIDS conditions as well as for the combined endpoint of opportunistic infections. The model included month of follow-up (restricted cubic splines with 4 knots at 1, 6, 24, and 60 months) and the following baseline covariates: CD4 cell count $(<200,200-299, \geq 300$ cells $/ \mu \mathrm{L})$, HIV RNA level $(<10,000,10,000-100,000,>100,000$ copies $/ \mathrm{mL})$, sex, acquisition group (heterosexual, homosexual/bisexual, injection drug use, other or unknown), calendar year (1998, 1999-2000, 20012003 , $\geq 2004)$, age $(<35,35-50,>50$ years), geographic origin (North America or Western Europe, Sub-Saharan Africa, other, or unknown), race (white, black, other, or unknown), years since HIV diagnosis $(<1,1-4, \geq 5$ years or unknown), whether or not the regimen was an NNRTI-based regimen, and cohort.

The variables we adjusted for in our models are associated with CPE score and widely known to be associated with the outcomes of interest. For example, a baseline CD4 cell count $<200$ cells $/ \mu \mathrm{L}$, a baseline HIV RNA $>100,000$ copies $/ \mathrm{mL}$, and a baseline age $>50$ years were associated with an increased odds of both HIV dementia and the combined endpoint of opportunistic infections compared with a baseline CD 4 cell count $\geq 300$ cells $/ \mu \mathrm{L}$, a baseline HIV RNA $<10,000$ copies $/ \mathrm{mL}$, and a baseline age $<35$ years, respectively (data not shown).

To adjust for potential selection bias due to infrequent followup, we computed inverse probability weights. Each patient in the above logistic models received a time-varying weight inversely proportional to the estimated probability of not being censored, for each month that patient was followed. ${ }^{28,29}$ We fit a pooled logistic model using the baseline covariates listed above, the baseline CPE score category, and the most recent measurement of the following timevarying covariates: $\mathrm{CD} 4$ cell count (restricted cubic spline with 5 knots at 10, 200, 350, 500, and 1,000 cells/ $\mu \mathrm{L}$ ), HIV RNA level $(<5,000,5,000-10,000,10,000-100,000,>100,000$ copies $/ \mathrm{mL})$, time since last laboratory measure $(0,1-2,3-4,5-6, \geq 7$ months), and AIDS (any category C AIDS-defining illness other than the neuroAIDS condition of interest), and estimated each patient's 


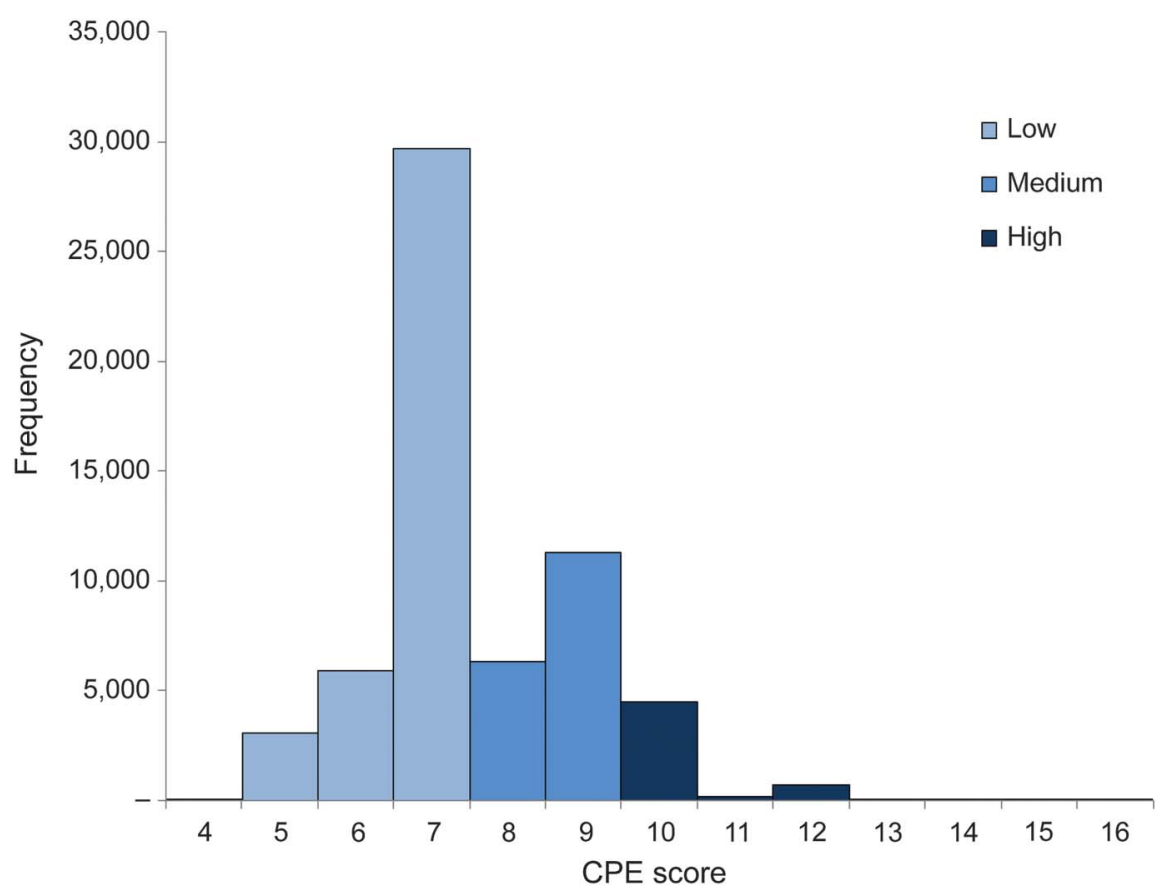

Number of individuals initiating treatment by CNS Penetration Effectiveness (CPE) score (range 4-16), HIV-CAUSAL Collaboration, 1998-2013.

probability of remaining uncensored in each month of follow-up. The models for the weights were fit before the final models. The weights were stabilized as described elsewhere ${ }^{28}$ and were then used to fit the final weighted regression model. The estimated weights for each of the 5 outcomes had mean 1.00 (first percentile: 0.96; 99th percentile: 1.20 ).

We used robust variance estimators that take into account the procedure of weight estimation to compute $95 \%$ confidence intervals (CIs) for each of our estimates. ${ }^{30}$ Under the assumption that the measured covariates are sufficient to adjust for confounding and selection bias, our approach emulates a nonblinded randomized trial in which patients were assigned to regimens with 1 of 3 CPE score categories. ${ }^{28}$

Several sensitivity analyses were performed for the outcome HIV dementia: we (1) varied the CPE score of regimens that were boosted with ritonavir because the CPE score category for boosted ritonavir is somewhat ambiguous in 2 regimens; (2) restricted the analysis to VACS in order to additionally adjust for the VACS Index ${ }^{31}$; (3) excluded VACS from the analysis; (4) estimated stabilized inverse probability weights to adjust for potential selection bias due to death, a competing risk ${ }^{32}$; (5) excluded cases of HIV dementia occurring in the first year; and (6) restricted the analysis to individuals who initiated therapy before the introduction of the first CPE scoring system in 2008. Exclusion of $0.15 \%$ of individuals with unusual treatment combinations (e.g., boosted nelfinavir and unboosted darunavir) did not materially affect the estimates. We also considered an alternative categorization of the CPE score by dividing the lowest category into 2 smaller categories ( $<6$ and 6 to $<8$ ), and estimated the average $\log$ hazard ratios using a model that included a flexible functional form for the continuous CPE score (restricted cubic spline with 4 knots at 5, 7, 9, and 12). We fit a pooled logistic model containing this form of the CPE score as well as the previously listed baseline covariates, and adjusted for potential selection bias due to infrequent follow-up as previously described.
All analyses were conducted with SAS 9.3 (SAS Institute, Cary, NC). The LOGISTIC procedure was used to fit the weighted regression models, and a nonparametric bootstrap with 500 samples was used to compute $95 \%$ CIs.

RESULTS A total of 61,938 individuals met the eligibility criteria for our study; 38,786 (62\%) initiated a regimen with a low CPE score, 17,687 (29\%) with a medium CPE score, and 5,465 (9\%) with a high CPE score. The mean score for individuals on an NNRTIbased regimen was 8.2 and the mean for individuals on a non-NNRTI-based regimen was 7.3. Table 1 shows the baseline characteristics of the study population by $\mathrm{CPE}$ score category. Individuals with a high CPE score were more likely to be female, heterosexual, initiating therapy before 2004, and of Sub-Saharan African origin. The median (interquartile range) follow-up time was $37(18,70)$ months.

During follow-up, there were 235 cases of HIV dementia, 169 cases of toxoplasmosis, 128 cases of cryptococcal meningitis, and 141 cases of progressive multifocal leukoencephalopathy. Forty individuals developed 2 of the 4 neuroAIDS conditions, and one individual developed 3. The incidence rate (per 10,000 person-years) was 9.0 for HIV dementia, 6.5 for toxoplasmosis, 4.9 for cryptococcal meningitis, and 5.4 for progressive multifocal leukoencephalopathy. The median (interquartile range) time from cART initiation to the event of interest was $14(2,39)$ months for HIV dementia, $4(1,16)$ months for toxoplasmosis, $10(1,25)$ months for cryptococcal 
Table 1 Baseline characteristics by CPE score category, HIV-CAUSAL Collaboration, 1998-2013

\begin{tabular}{|c|c|c|c|}
\hline \multirow[b]{2}{*}{ Baseline characteristics } & \multicolumn{3}{|l|}{ Persons, \% (n) } \\
\hline & $\begin{array}{l}\text { Low CPE score } \\
(\mathbf{n}=\mathbf{3 8}, 786)\end{array}$ & $\begin{array}{l}\text { Medium CPE score } \\
(\mathrm{n}=17,687)\end{array}$ & $\begin{array}{l}\text { High CPE score } \\
\text { (n=5,465) }\end{array}$ \\
\hline \multicolumn{4}{|l|}{ CD4 cell count, cells $/ \mu \mathrm{L}$} \\
\hline$<200$ & $37.8(14,674)$ & $44.9(7,939)$ & $43.5(2,377)$ \\
\hline 200 to $<300$ & $25.5(9,890)$ & $25.6(4,521)$ & $24.2(1,320)$ \\
\hline$\geq 300$ & $36.7(14,222)$ & $29.5(5,227)$ & $32.3(1,768)$ \\
\hline \multicolumn{4}{|l|}{ HIV RNA, copies/mL } \\
\hline$<10,000$ & $18.6(7,215)$ & $17.9(3,172)$ & $20.5(1,121)$ \\
\hline $10,000-100,000$ & $41.6(16,136)$ & $40.6(7,176)$ & $41.9(2,292)$ \\
\hline$>100,000$ & $39.8(15,435)$ & $41.5(7,339)$ & $37.6(2,052)$ \\
\hline \multicolumn{4}{|l|}{ Sex } \\
\hline Male & $80.0(31,016)$ & $74.4(13,157)$ & $66.2(3,618)$ \\
\hline Female & $20.0(7,770)$ & $25.6(4,530)$ & $33.8(1,847)$ \\
\hline \multicolumn{4}{|l|}{ Race } \\
\hline White & $23.9(9,285)$ & $23.3(4,125)$ & $17.8(970)$ \\
\hline Black & $13.9(5,373)$ & $20.0(3,541)$ & $21.9(1,198)$ \\
\hline Other & $62.2(24,128)$ & $56.7(10,021)$ & $60.3(3,297)$ \\
\hline \multicolumn{4}{|l|}{ Age, y } \\
\hline$<35$ & $34.6(13,418)$ & $35.5(6,280)$ & $41.9(2,289)$ \\
\hline $35-50$ & $47.4(18,382)$ & $47.1(8,327)$ & $43.6(2,384)$ \\
\hline$>50$ & $18.0(6,986)$ & $17.4(3,080)$ & 14.5 (792) \\
\hline \multicolumn{4}{|l|}{ Origin } \\
\hline North America or Western Europe & $60.3(23,373)$ & $54.7(9,671)$ & $53.1(2,903)$ \\
\hline Sub-Saharan Africa & $13.7(5,321)$ & $19.9(3,516)$ & $23.4(1,277)$ \\
\hline Other & $9.3(3,600)$ & $7.5(1,326)$ & 6.6 (359) \\
\hline Unknown & $16.7(6,492)$ & $17.9(3,147)$ & $16.9(926)$ \\
\hline \multicolumn{4}{|l|}{ Acquisition group } \\
\hline Heterosexual & $33.1(12,853)$ & $40.3(7,129)$ & $45.5(2,488)$ \\
\hline Homosexual/bisexual & $41.8(16,214)$ & $32.2(5,706)$ & $28.4(1,554)$ \\
\hline Injection drug user & $5.9(2,264)$ & $6.9(1,213)$ & 7.7 (418) \\
\hline Other/unknown & $19.2(7,455)$ & $20.6(3,639)$ & $18.4(1,005)$ \\
\hline \multicolumn{4}{|l|}{ Calendar year } \\
\hline 1998 & $7.4(2,865)$ & $7.2(1,283)$ & $6.0(326)$ \\
\hline $1999-2000$ & $8.5(3,291)$ & $14.6(2,578)$ & $29.1(1,593)$ \\
\hline 2001-2003 & $8.7(3,362)$ & $24.8(4,381)$ & $41.3(2,257)$ \\
\hline$\geq 2004$ & $75.4(29,268)$ & $53.4(9,445)$ & $23.6(1,289)$ \\
\hline \multicolumn{4}{|l|}{ Regimen } \\
\hline NNRTI-based & $47.2(18,295)$ & $59.4(10,501)$ & $74.6(4,079)$ \\
\hline non-NNRTI-based & $52.8(20,491)$ & $40.6(7,186)$ & $25.4(1,386)$ \\
\hline
\end{tabular}

Abbreviations: $\mathrm{CPE}=\mathrm{CNS}$ Penetration Effectiveness; NNRTI = nonnucleoside reverse transcriptase inhibitor.

meningitis, and $3(1,14)$ months for progressive multifocal leukoencephalopathy.

Table 2 shows the 3 most frequently used cART regimens with high, medium, and low CPE scores. Compared with initiating a cART regimen with a low
CPE score, the hazard ratio (95\% CI) for initiating a cART regimen with a high CPE score was 1.74 (1.15, 2.65) for HIV dementia, $0.90(0.50,1.62)$ for toxoplasmosis, $1.13(0.61,2.11)$ for cryptococcal meningitis, and $1.32(0.71,2.47)$ for progressive multifocal 


\begin{tabular}{|lcl|}
\hline Table 2 & $\begin{array}{l}\text { Most frequently used cART regimens with a low, medium, and high CPE } \\
\text { score, HIV-CAUSAL Collaboration, 1998-2013 }\end{array}$ \\
\hline Regimen & No. of initiators & CPE score (category) \\
Efavirenz, tenofovir, emtricitabine & 14,839 & 7 (low) \\
Nelfinavir, zidovudine, lamivudine & 3,368 & 7 (low) \\
Lopinavir, ritonavir, tenofovir, emtricitabine & 3,342 & 7 (low) \\
Efavirenz, zidovudine, lamivudine & 5,346 & 9 (medium) \\
Lopinavir, ritonavir, zidovudine, lamivudine & 3,823 & 9 (medium) \\
Efavirenz, lamivudine, abacavir & 1,837 & 8 (medium) \\
Nevirapine, zidovudine, lamivudine & 3,373 & 10 (high) \\
Indinavir, ritonavir, zidovudine, lamivudine & 757 & 10 (high) \\
Efavirenz, zidovudine, lamivudine, abacavir & 409 & 12 (high) \\
\hline
\end{tabular}

Abbreviations: $\mathrm{cART}=$ combined antiretroviral therapy; $\mathrm{CPE}=\mathrm{CNS}$ Penetration Effectiveness.
1.58). Figure 2 shows the $\log$ hazard ratio against the continuous CPE score for the combined endpoint of opportunistic infections and for HIV dementia (see figure e-1 on the Neurology ${ }^{\circledR}$ Web site at Neurology.org for the individual opportunistic infections).

The hazard ratio of HIV dementia for a high $\mathrm{CPE}$ score vs a low CPE score did not vary substantially by $\mathrm{CD} 4$ cell count, age, sex, and type of cART regimen (NNRTI-based vs non-NNRTI-based), but the 95\% CIs were wide (data not shown). The hazard ratios and $95 \%$ CIs did not change with different specifications of the functional form for month of follow-up (data not shown). None of the sensitivity analyses described in the previous section yielded appreciably different results (table e-1). Varying the cut points of the CPE score did not materially change the point estimates but resulted in wider CIs. Excluding cases of HIV dementia occurring in the first year of follow-up (table e-2) did not materially change the estimates of the effect

of CPE score on HIV dementia. leukoencephalopathy (table 3). Compared with a low CPE score, the respective hazard ratios $(95 \% \mathrm{CIs})$ for a medium CPE score were $1.01(0.73,1.39), 0.80$ $(0.56,1.15), 1.08(0.73,1.62)$, and $1.08(0.73$,

Table 3 Hazard ratios for CPE score, HIV-CAUSAL Collaboration, 1998-2013

\begin{tabular}{|c|c|c|c|c|c|c|}
\hline CPE score & Person-years & No. of events & $\begin{array}{l}\text { Unadjusted } \\
\text { hazard ratio }\end{array}$ & $95 \% \mathrm{Cl}$ & $\begin{array}{l}\text { Adjusted } \\
\text { hazard ratio }\end{array}$ & $95 \% \mathrm{Cl}$ \\
\hline \multicolumn{7}{|c|}{ HIV dementia } \\
\hline Low & 140,962 & 127 & 1.00 & Reference & 1.00 & Reference \\
\hline Medium & 86,799 & 72 & 0.97 & $0.72,1.30$ & 1.01 & $0.73,1.39$ \\
\hline High & 32,097 & 36 & 1.55 & $1.06,2.26$ & 1.74 & $1.15,2.65$ \\
\hline \multicolumn{7}{|c|}{ Opportunistic infections ${ }^{b}$} \\
\hline Low & 140,553 & 245 & 1.00 & Reference & 1.00 & Reference \\
\hline Medium & 86,455 & 134 & 1.09 & $0.88,1.34$ & 0.99 & $0.80,1.22$ \\
\hline High & 31,985 & 49 & 1.18 & $0.87,1.62$ & 1.08 & $0.77,1.52$ \\
\hline \multicolumn{7}{|c|}{ Toxoplasmosis } \\
\hline Low & 140,983 & 106 & 1.00 & Reference & 1.00 & Reference \\
\hline Medium & 86,807 & 45 & 0.86 & $0.60,1.22$ & 0.80 & $0.56,1.15$ \\
\hline High & 32,099 & 18 & 0.94 & $0.57,1.57$ & 0.90 & $0.50,1.62$ \\
\hline \multicolumn{7}{|c|}{ Cryptococcal meningitis } \\
\hline Low & 141,098 & 64 & 1.00 & Reference & 1.00 & Reference \\
\hline Medium & 86,818 & 48 & 1.35 & $0.92,1.98$ & 1.08 & $0.73,1.62$ \\
\hline High & 32,121 & 16 & 1.43 & $0.83,2.48$ & 1.13 & $0.61,2.11$ \\
\hline \multicolumn{7}{|c|}{$\begin{array}{l}\text { Progressive multifocal } \\
\text { leukoencephalopathy }\end{array}$} \\
\hline Low & 141,109 & 81 & 1.00 & Reference & 1.00 & Reference \\
\hline Medium & 86,849 & 43 & 1.12 & $0.77,1.64$ & 1.08 & $0.73,1.58$ \\
\hline High & 32,116 & 17 & 1.36 & $0.80,2.33$ & 1.32 & $0.71,2.47$ \\
\hline
\end{tabular}

Abbreviations: $\mathrm{Cl}=$ confidence interval; $\mathrm{CPE}=\mathrm{CNS}$ Penetration Effectiveness.

${ }^{a}$ Adjusted for cohort, month of follow-up, baseline CD4 cell count, baseline HIV RNA level, sex, acquisition group, calendar year, age, geographic origin, race, years since HIV infection, and type of drug regimen, as well as time-varying CD4 cell count, RNA level, time since last measurement, and AIDS. Stabilized inverse probability weights were used to account for censoring due to infrequent follow-up.

${ }^{\mathrm{b}}$ Includes toxoplasmosis, cryptococcal meningitis, and progressive multifocal leukoencephalopathy. 
Figure 2 Estimated log hazard ratios and $95 \%$ confidence intervals

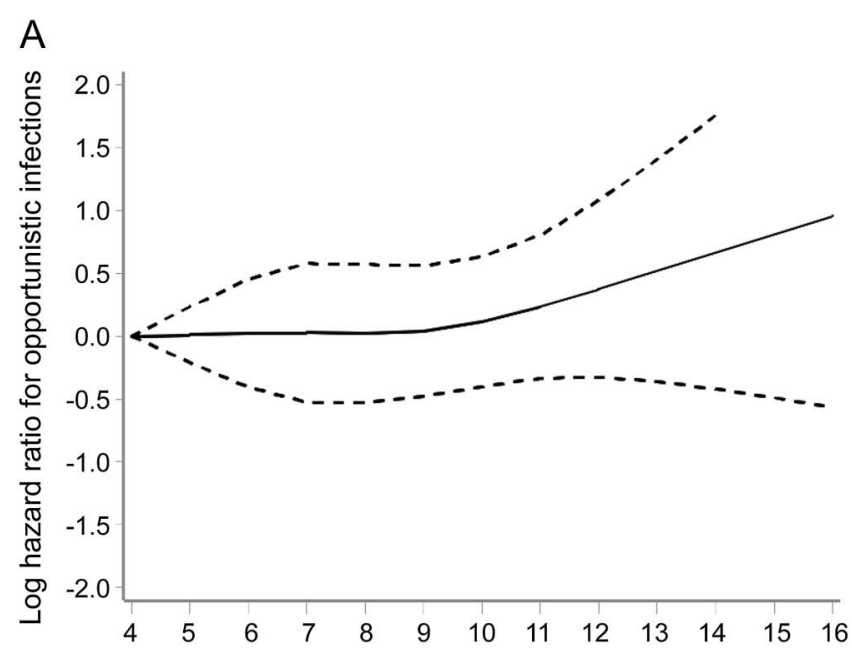

B

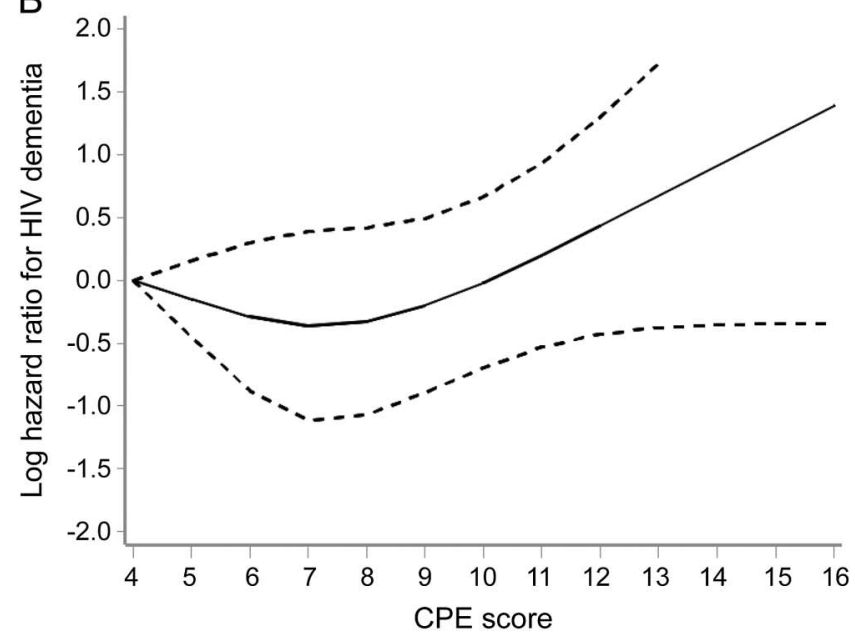

Estimated log hazard ratios and 95\% confidence intervals for opportunistic infections (A) (toxoplasmosis, cryptococcal meningitis, or progressive multifocal leukoencephalopathy) and HIV dementia (B) comparing each CNS Penetration Effectiveness (CPE) score with a CPE score of 4 (lowest), HIV-CAUSAL Collaboration, 1998-2013.

DISCUSSION We estimated that the incidence of HIV dementia increases by more than $70 \%$ after initiating an antiretroviral regimen with a high $\mathrm{CPE}$ score compared with a low score. However, we found little change in the incidence of toxoplasmosis, cryptococcal meningitis, and progressive multifocal leukoencephalopathy. These results are unexpected, and the interpretation of our effect estimates needs to be tempered by the limitations of our study.

In the only published study that has considered CPE scores in relation to neuroAIDS among HIVpositive individuals, the incidence of neuroAIDS was similar for a baseline CPE score of 10 or greater and for a baseline CPE score of 4 or less, but the small number of events resulted in a wide $95 \% \mathrm{CI}$ (hazard ratio: 0.95; 95\% CI: 0.53, 1.72). Furthermore, the study was not restricted to antiretroviral therapy-naive individuals and effect estimates for individual neuroAIDS conditions such as HIV dementia were not provided. ${ }^{24}$ Other studies of the association between CPE scores and risk of cognitive impairment (but not neuroAIDS) have had conflicting results. ${ }^{17,19-21,33}$

Our findings that CPE score does not affect the incidence of opportunistic infections may not be particularly surprising, because the development of these neuroAIDS conditions may be very closely connected to the degree of impaired cell-mediated immunity and not associated with antiretroviral penetration. ${ }^{4,5}$ In contrast, antiretroviral penetration into the brain may lead to deposition of $\beta$-amyloid plaques, which has been proposed as a possible explanation for a harmful effect of high CPE score on HIV dementia. ${ }^{34}$ One study observed a higher percentage of extracellular $\beta$-amyloid in cART-treated patients than in untreated HIV-positive individuals, ${ }^{35}$ and HIV-positive individuals with HIV-associated dementia have higher levels of intraneuronal $\beta$-amyloid immunoreactivity compared with HIV-positive individuals without HIVassociated dementia. ${ }^{34-36}$ However, the underlying mechanism through which antiretroviral penetration could cause HIV dementia remains unknown. The hypothesis that antiretroviral penetration increases the incidence of HIV dementia via deposition of $\beta$-amyloid plaques requires further research to determine whether these associations are in fact causal. Alternative pathways including antiretroviral-related direct neuronal damage and mitochondrial toxicity should also be evaluated. ${ }^{15}$

Another explanation for the higher dementia risk for regimens with a high CPE score is that these regimens are less effective to treat HIV disease, for example, because of incomplete adherence: 68\% of individuals in the study deviated from their initial regimen at some point. However, both the high average proportion of follow-up spent on the initial regimen (58\%) and the lack of a strong association between CPE score and opportunistic infections do not support this explanation.

Similar to any other observational study, the validity of our estimates relies on the untestable assumption that the measured covariates were sufficient to adjust for confounding and selection bias. It is possible that consideration of CPE scores is a factor for decisions concerning antiretroviral regimens in patients with neurocognitive symptoms. If individuals with neurocognitive symptoms are more likely to initiate antiretroviral regimens with higher CPE scores, the estimated effect on dementia might be explained by this confounding by indication. If this were the case, we would expect the estimated effect on dementia to disappear or to attenuate after a certain amount of time. However, excluding cases of HIV dementia occurring in the first year of follow-up does not materially change the results. Furthermore, restricting the 
analysis to individuals who initiated therapy before the introduction of the first CPE scoring system in 2008 does not affect the results.

Some limitations of our study should be noted. First, with relatively few events, the 95\% CIs around our effect estimates are wide. However, our study is the largest one to date on this topic. Second, the cohorts included in this analysis are from developed countries; our results may not be generalizable to resource-limited settings or to other health care systems. Third, with the exception of VACS, the contributing cohorts used diagnostic procedures that reflect standard clinical practice in Europe. Excluding VACS from the analysis, however, did not significantly alter the results. Fourth, while our effect estimates are adjusted for cohort, we were not able to adjust for the individual centers within each cohort. Thus, some residual confounding due to centers within each cohort is theoretically possible. Finally, the average duration of follow-up in our study was approximately 3 years. Future studies will be needed to investigate the effect of antiretroviral penetration on the long-term incidence of neuroAIDS, as well as the effect of newer antiretrovirals that are not well represented in current studies, including ours.

We estimated that initiation of a cART regimen with a high CPE score increases the risk of HIV dementia, but not of other neuroAIDS conditions. These findings should be interpreted cautiously, and additional studies are needed to examine the effect of CPE score on the incidence of HIV dementia more closely. Together with additional data on the safety and effectiveness of different cART regimens, these results may be useful to plan the management of individuals with HIV infection.

\section{AUTHOR AFFILIATIONS}

From the Harvard School of Public Health (E.C.C., L.E.C., R.L., M.H.), Boston, MA; Yale School of Medicine (A.J., J.T.), Yale School of Public Health (A.J.), New Haven, CT; University College London (C.S.); Imperial College HealthCare NHS Trust (A.W.), London, UK; Stichting HIV Monitoring (A.v.S.), Amsterdam, the Netherlands; Hospital Clinic Universitari (J.M.), Barcelona; University Hospital of Bellvitge (D.P.), Barcelona, Spain; MRC Clinical Trials Unit (A.O.), London, UK; Hospital Universitario La Paz (J.A.), Madrid; Hospital Ramón y Cajal (S.M.), Madrid, Spain; Center for Research in Epidemiology and Population Health (L.M.), Paris, France; Centro Sanitario Sandoval (J.d.R.), Madrid, Spain; Université Victor Segalen Bordeaux 2 (F.D.), France; University Hospital Basel (H.B.); Institute of Social and Preventive Medicine (G.W.), Bern, Switzerland; University of Athens (G.V.), Greece; Evaggelismos Athens General Hospital (A.S.), Greece; Institut Gustave Roussy (E.L.), Villejuif; Bicêtre Hospital (J.G.), Paris; and Institut national de la santé et de la recherche médicale (INSERM) (D.C.), Paris, France.

\section{AUTHOR CONTRIBUTIONS}

E.C. Caniglia, L.E. Cain, and M.A. Hernán conceived the study and wrote the first draft of the manuscript. E.C. Caniglia completed the literature search and created the figures. All authors contributed to the final draft. A. Justice, J. Tate, R. Logan, C. Sabin, A. Winston, A. van Sighem, J. Miro, D Podzamczer, A. Olson, J. Arribas, S. Moreno, L. Meyer, J. del Romero, F. Dabis, H. Bucher, G. Wandeler, G. Vourli, A. Skoutelis, E. Lanoy, J. Gasnault, and D. Costagliola contributed to data collection.
E.C. Caniglia and L.E. Cain completed the analyses and data interpretation with assistance and advice from M.A. Hernán and R. Logan. Members of the Writing Committee include Ellen C. Caniglia, Lauren E. Cain, PhD (Coordinating Center), Amy Justice, PhD, Janet Tate, PhD (VACS), Roger Logan, $\mathrm{PhD}$ (Coordinating Center), Caroline Sabin, PhD, Alan Winston, MD (UK CHIC), Ard van Sighem, PhD (ATHENA), Jose M. Miro, PhD, Daniel Podzamczer, PhD (PISCIS), Ashley Olson, PhD (UKREG), José Ramón Arribas, MD, Santiago Moreno, PhD (CoRIS/CoRIS-MD), Laurence Meyer, PhD (PRIMO/SEROCO), Jorge del Romero, MD (GEMES), François Dabis, PhD (Aquitaine), Heiner C. Bucher, MD, Gilles Wandeler, MD (SHCS), Georgia Vourli, MSc, Athanasios Skoutelis, MD (AMACS), Emilie Lanoy, PhD, Jacques Gasnault, MD, Dominique Costagliola, PhD (FHDH-ANRS C04), and Miguel A. Hernán, DrPH (Coordinating Center).

\section{ACKNOWLEDGMENT}

The authors thank Fabrice Bonnet for his comments on an earlier version of the manuscript.

\section{STUDY FUNDING}

This study has been financed in the framework of the HIV-CAUSAL Collaboration, supported by NIH grant R01-AI102634. E.C. Caniglia was supported by NIH grant T32-AI007535. The funders had no role in study design, data collection and analysis, decision to publish, or preparation of the manuscript.

\section{DISCLOSURE}

E. Caniglia, L. Cain, A. Justice, J. Tate, and R. Logan report no disclosures relevant to the manuscript. C. Sabin has received travel grants, fees for speaking and honoraria from various pharmaceutical companies including Bristol-Myers Squibb (BMS), Gilead Sciences, Boehringer Ingelheim, Janssen Pharmaceutica, and Tibotec. A. Winston has received honoraria or research grants, or been a consultant or investigator, in clinical trials sponsored by Abbott, Boehringer Ingelheim, BMS, Gilead Sciences, GlaxoSmithKline (GSK), Janssen Cilag, Roche, Pfizer, and ViiV Healthcare. A. van Sighem reports no disclosures relevant to the manuscript. J. Miro has received honoraria for consultant and/or research grants from Abbott, Boehringer Ingelheim, BMS, Cubist, Novartis, GSK, Gilead Sciences, Pfizer, Roche, Theravance, and ViiV. D. Podzamczer has received research grants and/or honoraria for advisories and/or conferences from Boehringer Ingelheim, GSK, ViiV, Pfizer, BMS, Abbott, Gilead, Janssen, and Merck. A. Olson, J. Arribas, and S. Moreno report no disclosures relevant to the manuscript. L. Meyer has received honoraria from GSK. J. del Romero and F. Dabis report no disclosures relevant to the manuscript. H. Bucher has received travel grants, honoraria, and unrestricted research grants from GSK, BMS, Gilead, Roche, Abbott, Tibotec, Janssen, Boehringer Ingelheim, and ViiV Healthcare. G. Wandeler, G. Vourli, A. Skoutelis, E. Lanoy, and J. Gasnault report no disclosures relevant to the manuscript. D. Costagliola has received travel grants, consultancy fees, honoraria, or study grants from various pharmaceutical companies including Abbott, Boehringer Ingelheim, BMS, Gilead Sciences, GSK, Janssen, Merck, and Roche. M. Hernán reports no disclosures relevant to the manuscript. Go to Neurology.org for full disclosures.

Received August 15, 2013. Accepted in final form February 11, 2014.

\section{REFERENCES}

1. Wright E. Neurological complications of long-term treated HIV disease: what's on your mind? Presented at the 19th Conference on Retroviruses and Opportunistic Infections (CROI); Seattle; 2012.

2. Sacktor N. The epidemiology of human immunodeficiency virus-associated neurological disease in the era of highly active antiretroviral therapy. J Neurovirol 2002;8 (suppl 2):115-121.

3. Heaton R, Clifford D, Frankin DJ, et al. HIV-associated neurocognitive disorders persist in the era of potent antiretroviral therapy: CHARTER Study. Neurology 2010;75: 2087-2096. 
4. Sacktor N, Lyles R, Skolasky R, et al. HIV-associated neurologic disease incidence changes: Multicenter AIDS Cohort Study, 1990-1998. Neurology 2001;56:257260.

5. d'Arminio Monforte A, Cinque P, Mocroft A, et al. Changing incidence of central nervous system diseases in the EuroSIDA cohort. Ann Neurol 2004;55:320-328.

6. Engsig F, Hansen A, Omland L, et al. Incidence, clinical presentation, and outcome of progressive multifocal leukoencephalopathy in HIV-infected patients during the highly active antiretroviral therapy era: a nationwide cohort study. J Infect Dis 2009;199:77-83.

7. Khanna N, Elzi L, Mueller N, et al. Incidence and outcome of progressive multifocal leukoencephalopathy over 20 years of the Swiss HIV Cohort Study. Clin Infect Dis 2009;48:1459-1466.

8. Garvey L, Winston A, Walsh J, et al; UK Collaborative HIV Cohort (CHIC) Study Steering Committee. HIVassociated central nervous system diseases in the recent combination antiretroviral therapy era. Eur J Neurol 2011;18:527-534.

9. Lescure F, Omland L, Engsig F, et al. Incidence and Impact on mortality of severe neurocognitive disorders in persons with and without HIV infection: a Danish nationwide cohort study. Clin Infect Dis 2011;52:235-243.

10. Robertson K, Su Z, Margolis D, et al. Neurocognitive effects of treatment interruption in stable HIV-positive patients in an observational cohort. Neurology 2010;74: 1260-1266.

11. Mothobi N, Brew B. Neurocognitive dysfunction in the highly active antiretroviral therapy era. Curr Opin Infect Dis 2012;25:4-9.

12. Liner KJ, Ro MJ, Robertson K. HIV, antiretroviral therapies, and the brain. Curr HIV/AIDS Rep 2010;7: 85-91.

13. Letendre S, Marquie-Beck J, Capparelli E, et al. Validation of the CNS penetration-effectiveness rank for quantifying antiretroviral penetration into the central nervous system. Arch Neurol 2008;65:65-70.

14. Letendre S. Background and rationale of the CPE score. Presented at the 2nd International Workshop on HIV \& Aging. Baltimore; 2011.

15. Robertson K, Liner J, Meeker R. Antiretroviral neurotoxicity. J Neurovirol 2012;18:388-399.

16. Letendre S. Central nervous system complications in HIV disease: HIV-associated neurocognitive disorder. Top Antivir Med 2011;19:137-142.

17. Marra C, Zhao Y, Clifford D, et al. Impact of combination antiretroviral therapy on cerebrospinal fluid HIV RNA and neurocognitive performance. AIDS 2009;23:1359-1366.

18. Cusini A, Vernazza P, Yerly S, et al. Higher CNS penetration-effectiveness of long-term combination antiretroviral therapy is associated with better HIV-1 viral suppression in cerebrospinal fluid. J Acquir Immune Defic Syndr 2013;62:28-35.

19. Cysique L, Vaida F, Letendre S, et al. Dynamics of cognitive change in impaired HIV-positive patients initiating antiretroviral therapy. Neurology 2009;73:342-348.

20. Tozzi V, Balestra P, Salvatori M, et al. Changes in cognition during antiretroviral therapy: comparison of 2 different ranking systems to measure antiretroviral drug efficacy on HIV-associated neurocognitive disorders. J Acquir Immune Defic Syndr 2009;52:56-63.

21. Ciccarelli N, Fabbiani M, Di Giambenedetto S, et al. Efavirenz associated with cognitive disorders in otherwise asymptomatic HIV-infected patients. Neurology 2011; 76:1403-1409.

22. Lanoy E, Guiguet M, Bentata $M$, et al. Survival after neuroAIDS: association with antiretroviral CNS penetrationeffectiveness score. Neurology 2011;76:644-651.

23. Ellis R, Letendre S, Vaida F, et al. A randomized, controlled trial of a central nervous system-targeted ART strategy for HIV-associated neurocognitive disorders. Presented at the 20th Conference on Retroviruses and Opportunistic Infections. Atlanta; 2013.

24. Garvey L, Winston A, Walsh J, et al. Antiretroviral therapy CNS penetration and HIV-1-associated CNS disease. Neurology 2011;76:693-700.

25. 1993 revised classification system for HIV infection and expanded surveillance case definition for AIDS among adolescents and adults. MMWR Recomm Rep 1992;41:1-9.

26. Ray M, Logan R, Sterne JA, et al; HIV-CAUSAL Collaboration. The effect of combined antiretroviral therapy on overall mortality of HIV-infected individuals. AIDS 2010; 24:123-137.

27. D’Agostino R, Lee M, Belanger A, Cupples L, Anderson K, Kannel W. Relation of pooled logistic regression to time dependent Cox regression analysis: the Framingham Heart Study. Stat Med 1990;9:1501-1515.

28. Hernán M, Brumback B, Robins J. Marginal structural models to estimate the causal effect of zidovudine on the survival of HIV-positive men. Epidemiology 2000;11: 561-570.

29. Sterne J, Hernan M, Ledergerber B, et al. Long-term effectiveness of potent antiretroviral therapy in preventing AIDS and death: a prospective cohort study. Lancet 2005;366:378-384.

30. Robins J. Marginal structural models. In: 1997 Proceedings of the American Statistical Association, Section on Bayesian Statistical Science, Alexandria, VA. 1998:1-10.

31. Tate J, Justice A, Hughes M, et al. An internationally generalizable risk index for mortality after one year of antiretroviral therapy. AIDS 2013;27:563-572.

32. Lau B, Cole S, Gange S. Competing risk regression models for epidemiologic data. Am J Epidemiol 2009;170:244-256.

33. Ciccarelli N, Fabbiani M, Colafigli M, et al. Revised central nervous system neuropenetration-effectiveness score is associated with cognitive disorders in HIV-infected patients with controlled plasma viraemia. Antivir Ther 2013;18:153-160.

34. Giunta B, Ehrhart J, Obregon D, et al. Antiretroviral medications disrupt microglial phagocytosis of $\beta$-amyloid and increase its production by neurons: implications for HIVassociated neurocognitive disorders. Mol Brain 2011;4:4-23.

35. Xu J, Ikezu T. The comorbidity of HIV-associated neurocognitive disorders and Alzheimer's disease: a foreseeable medical challenge in post-HAART era. J Neuroimmune Pharmacol 2009;4:200-212.

36. Achim C, Adame A, Dumaop W, Everall I, Masliah E; Neurobehavioral Research Center. Increased accumulation of intraneuronal amyloid $\beta$ in HIV-infected patients. J Neuroimmune Pharmacol 2009;4:190-199. 